\title{
Communication managériale et désaffiliation
}

In: Quaderni. N. 53, Hiver 2003/2004. La convivialité, fable contemporaine. pp. 81-91.

Citer ce document / Cite this document :

Palmade Jacqueline. Communication managériale et désaffiliation. In: Quaderni. N. 53, Hiver 2003/2004. La convivialité, fable contemporaine. pp. 81-91.

doi : 10.3406/quad.2003.1599

http://www.persee.fr/web/revues/home/prescript/article/quad_0987-1381_2003_num_53_1_1599 


\section{Dossier}

communication managériale et désaffiliation

\section{Jacqueline Palmade}

\author{
Professeur émérite de Sociologie \\ Université Paris IX Dauphine
}

Aujourd'hui, lorsqu' un cadre arrive le matin dans son bureau, son premier geste est (doit être) de consulter sa messagerie. L'ordinateur lui dit : bonjour, bonne journée ; simultanément, il lui indique la valeur boursière de son entreprise et des entreprises concurrentes; lorsqu'un ouvrier arrive dans son atelier, avant de prendre son poste, l'écran d'affichage lui indique : le taux des accidents du travail, le taux d'absentéisme, le taux de pièces défectueuses, le volume de production, le coût des activités improductives (pauses, électricité, réparations, photocopies...), le classement de son atelier par rapport aux autres, l'objectif à atteindre.

Tout en étant informé, en temps réel, de ces aspects de la vie de son entreprise, chacun appréhende d'apprendre, par la rumeur, que son chef a encore changé, qu'une fusion ou une restructuration est en cours, qu'il y aura des changements d'affectation d'emplois (dans un autre site, une autre entreprise, en sous-traitance), qu'il lui faudra se reconvertir, qu'une politique d'externalisation est en cours, qu'il y aura des réductions de personnel.

La distance entre l'image de l'entreprise que tente de créer la communication (externe et interne) de l'entreprise et la réalité - objective et vécue - des conditions de travail et d'emploi, relève plus d'un clivage entre la logique économique et stratégique portée par la hiérarchie supérieure et la logique existentielle de l'exécution qu'elle n'est l'expression d'une utopie qui pourrait devenir projet collectif.

La communication, notamment dans sa composante de convivialité, se doit de résoudre 
ces contradictions, du moins de les effacer, de les dénier, ou de les ériger en norme de la modernité, plus précisément en norme de l'économie néolibérale.

Depuis 1970 , on observe un investissement de plus en plus important des entreprises, y compris des entreprises publiques, dans une politique de communication, politique dont la finalité est d'accompagner les réformes structurelles obligées par le marché, de les faire accepter en déplaçant le niveau structural (notamment celui des relations sociales de travail) sur le niveau culturel (au singulier). Simultanément, notamment depuis vingt ans, on observe une augmentation significative d'un processus de précarisation/ prolétarisation des salariés de l'exécution et de l'encadrement moyen, évolution corrélative d'une augmentation d'un clivage entre l'encadrement supérieur et l'exécution. Cette dualisation se caractérise, pour l'encadrement supérieur, par un investissement stratégique aux orientations néolibérales de la société (dont ils profitent), pour l'exécution, par une prolétarisation/précarisation dont le risque de perdre l'emploi, la déqualification, l'affaiblissement de l'appartenance à un collectif de travail, la perte de la valeur normative, expressive et socialisatrice du travail sont responsables (qu'ils subissent).

À cette évolution correspondrait l'émergence, relativement récente, d'une politique de communication centréc sur la confiance et l'éthique.

\section{Les promesses du discours managérial}

Dans l'immédiat après-guerre, la communication dans les organisations était mise en œuvre dans et par des actions de formation aux relations humaines inspirées de la recherche-action réalisée à la Western-Electric en 1938 et dans la formation à l'art du commandement. À cette même époque, Herbert Simon (1945) proposait à des fins de rationalité et d'efficacité du système, la nécessité de mettre en cuvre une politique de communication visant à faire adhérer chaque membre de l'organisation à ses normes et à ses valcurs sans pour autant que leur origine, leur justification soient légitimées.

Ce n'est que beaucoup plus tard, vers 1965-70, que la communication deviendra une sorte d'entité existant en quelque sortc par elle-même, autonomisće des autres fonctions de l'organisation, communication qui aura alors un caractère soit technocratique soit d'idćalité. Cette dualité lui confère de faire passer en arrière-plan la réalité vécue des rapports et des interactions structurées par (et dans) le travail et son organisation, son management. C'est le versant d'idéalité qui entretient des proximités avec la convivialité. À l'extrême, la communication devient en sa nature échange d'information (de signaux) sur ce que chacun est découvreur de ce qu'il vit et de ce qui pour lui et d'une même manière sera découvert comme étant vécu par les autres. Le travail et la création de sens de cette double découverte sont ainsi objectivés, réifiés. Dans cette perspective, on communique sur ce qui est posé comme étant vécu. Par contre, l'engagement où l'on est pris dans des rapports d'engagement - qui sont cux-mêmes le plus fondateur d'un engagement - deviennent comme des objets pour le dire des communiquants. Ce dire des "communiquants", idéalisé, cst alors pris comme 
substitut des conduites vécues. Cette idéalité de la communication oriente, complémentairement à une communication technocratique, la communication stratégique au seul service du néo-management.

Les éléments de persuasion, de mobilisation, d'illusion d'alliance de travail, de découverte de sens, font appel à des ressorts psychologiques et idéologiques : plaisir, identification héroïque, agressivité conquérante, toute-puissance, négation des différences notamment des inégalités, fusion dans la communauté unifiée et totalisante, déplacement de la valeur travail sur l'organisation, mise à distance de l'autorité, réduction ou maîtrise de l'incertitude.

La convivialité qui n'est jamais explicitement nommée, mais dont le sens oriente la communication managériale, devient promesse de relations a-conflictuelles, d'affiliation, de protection contre sa propre violence, de soutien à l'anxiété de l'incertitude. Le caractère "convivial" de cette communication imaginaire, "managinaire" (de Gaulejac), devient une communication amputée de la fonction symbolique de la parole. "Il y a de plus en plus d'experts en communication dans notre entreprise mais on a de moins en moins le temps - le droit - de parler", disent les salariés de l'exécution d'une grande entreprise lors d'une recherche évaluant deux ans de politique de communication (1993).

Rappelons que si le sens de la convivialité est de faire partager le festin aux invités devenus convives, il leur est interdit de "cracher dans la soupe".
Ėve Chiapello (1999), à partir de l'analyse de deux corpus d'ouvrages de management destinés aux cadres (un premier de 60 textes écrits par 48 auteurs entre 1959 et 1968, un deuxième de 60 textes écrits par 40 auteurs entre 1989 et 1994), a mis en évidence l'évolution de la problématique du management. Pour la première période, $40 \%$ des textes sont américains et $66 \%$ français ; pour la deuxième période, $17 \%$ des textes sont américains et $73 \%$ français. L'évolution de l'origine culturelle des corpus est significative de l'hégémonie du modèle référentiel néo-libéral.

Une première remarque concerne la grande homogénéité des textes concernant notamment les exemples et les références théoriques. Ils sont comme les mêmes d'une même religion. Les références, généralement philosophiques ou religieuses, donnent aux discours un caractère normatif, dogmatique. Elles sont là comme figures emblématiques qui cautionnent la validité des discours, les légitiment. Ainsi, défilent comme des fétiches : la Bible, le bouddhisme, Socrate, Platon, Sénèque, Montaigne, Kant... Habermas.

Les éléments les plus significatifs des thèmes qui rendent compte de l'évolution entre 1960 et 1970 du discours managérial concernent essentiellement : l'abandon de la direction par objectifs, de la méritocratie, de la valorisation des cadres comme nouveau corps social responsable de la croissance; la valorisation d'une organisation en réseau à structure matricielle ou adhocratique, réseau où chaque élément du système, grâce à l'informatisation, est interconnecté ; la conception d'une organisation où la rationalité devient vision créatrice, où le rapport à l'entreprise devient contrat librement 
consenti, où la méritocratie devient développement personnel, où le chef devient animateur, coordinateur, où les ouvriers deviennent des opérateurs, des partenaires ou des collaborateurs, où le contrôle devient autocontrôle ; la défiance, confiance.

Ce système, tenu d'une main invisible par la rationalité technocratique et la hiérarchie, serait régulé, auto-régulé, car le destinateur-destinataire du travail "le capital" serait devenu le "clientfournisseur", et le conflit sur le sens du travail partage de sens.

L'analyse de l'apparition et de l'évolution du nombre des occurrences présentes dans les deux corpus entre les deux périodes considérées est très significative des idéologies qui sous-tendent le néo-management. Nous avons retenu les plus significatives (voir tableau ci-après).

Il reste à la communication à vendre les promesses d'absence de conflits structurels et relationnels, la disparition de l'autorité et du contrôle, le développement personnel, l'accès au sens.

Les auteurs de ces ouvrages distribuent, en "bons" et "mauvais" objets, les catégories à partir desquelles ils organisent leurs discours. Sont systématiquement regroupés en mauvais objets, la "bureaucratie" et le couple "domination/ aliénation"; réalités relevant pour ces autcurs d'un passé révolu.

Anne Salmon (2002), à partir d'un corpus constitué de textes d'entreprises, de textes de consultants et de professionnels, de rapports d'experts, de colloques et revues sur l'éthique, analyse tant sur le plan de sa forme que de son contenu le thème de l'éthique, thème issu des USA et dont l'ampleur croît au cours des années 1990. Cet auteur montre le caractère instrumental de la sphère éthique à des fins de remobilisation des salariés aux objectifs et finalités de l'Entreprise. Anne Salmon montre que, si les motifs idéels ne sont plus puisés dans les sphères religieuses et culturelles - c'est-à-dire dans des sphères extérieures à la sphère économique -, mais dans la sphère économique qu'ils doivent servir, cette absence de tiers symbolique - transcendant transforme la communication qui les énonce et les justifie en caractère tautistique (totalitaire, autistique - Sfez). L'offre éthique qui scrait ainsi produite par l'ordre économique proposerait un modèle d'être au travail inspiré de "la communication publicitaire selon lequel la tension entre désir et raison serait déniée": la contrainte deviendrait spontanćité ; le formel, l'informel ; la communication, expression individuelle ; le commandement, leadership ; le leadership, révélation de l'autre; l'éthique, authenticité.

L'éthique économique deviendrait moyen de régulation sans horizon extéricur à elle-même, elle ne donnerait plus lc "sens-direction", mais donnerait des "valeurs-croyance", épargnant alors le doute généralisé, l'angoisse éthique.

La promesse d'un plaisir partagé, la promesse d'une toute-puissance qui supprime la quête du sens tout en s'emparant du sens, rapprocherait l'éthique économique des promesses d'une société conviviale où l'homme contrôlerait l'inconnu du dépassement des rapports sociaux qui structurent le rapport au travail et son 


\section{Occurrences}

$\begin{array}{llc} & \mathbf{1 9 6 0} & \mathbf{1 9 9 0} \\ \text { Cadres } & 986 & 219 \\ \text { Dirigeants } & 724 & 369 \\ \text { Subordonnés } & 797 & 343\end{array}$

\begin{tabular}{lll}
\hline Direction & 549 & 0 \\
Chef & 487 & 0 \\
Autorité & 316 & 0 \\
Organisation & 343 & 0 \\
\hline
\end{tabular}

\begin{tabular}{lrr}
\hline Syndicat & 83 & 0 \\
Capital & 190 & 0 \\
Client-fournisseur & 38 & 363 \\
\hline
\end{tabular}

\begin{tabular}{lll}
\hline Réseau & 0 & 450 \\
Équipe & 0 & 392 \\
Projet & 0 & 375 \\
Manager & 0 & 299 \\
\hline
\end{tabular}

$\begin{array}{lll}\text { Développement } & 0 & 213\end{array}$

Vie $\quad 0 \quad 205$

Sens $\quad 0 \quad 188$

$\begin{array}{lll}\text { Monde } & 0 & 175\end{array}$

$\begin{array}{lll}\text { Meilleur, communicateur } & 0 & 40\end{array}$

\begin{tabular}{lll}
\hline L'Homme & 173 & disparu \\
\hline
\end{tabular}

management.

Ces orientations managćrialcs nc sont pas nées ex nihilo, elles apparaissent dans un contexte socio-économique et culturel sur lequel à la fois elles s'appuient et dont elles sont le symptôme. Au niveau socio-économique, nous évoquerons : les transformations de l'emploi (moins d'ouvriers, plus d'employés, plus de cadres, plus d'accès des femmes au travail); la dćconstruction du monde du travail ; l'affaiblissement des défenses du monde du travail ; la crise du capitalisme. Au niveau culturel, nous évoquerons : la montée de l'individualisme, l'importance accordée à la vie 
hors travail (loisirs, sports), la médiatisation généralisée de la socićté qui métamorphose toute activité sociale (y compris le travail) en objet de consommation, la prégnance des sphères du Pouvoir et de l'Avoir sur la sphère du Valoir.

\section{La confrontation à la réalité}

\section{Le cas des bureaux-paysagers}

La conception de l'aménagement des espaces de travail en "bureaux-paysagers" est un idćal-type du clivage entre le discours emblématique de la convivialité au travail qu'clle favoriserait déterminerait-, et les objectifs d'efficacité et/ou de productivité visés par cette nouvelle production des espaces de travail. Le décloisonnement des espaces donnerait à voir l'abandon de la conception traditionnelle selon laquelle la hiérarchie des fonctions et des statuts des personnes était incorporée dans l'aménagement des espaces. Aujourd'hui, chacun, en partageant le même espace deviendrait alors, du fait de cette spatialisation ouverte, non structurée, anonyme, l'égal de l'autre. Seule la haute hiérarchie a droit à des bureaux fermés donnant sur l'extérieur par des fenêtres. C'est le cas du siège social "challenger" de Bouygues à St Quentin-en-Yvelines. Ainsi, l'effacement des repères spatiaux de la hiérarchie, notamment entre les cadres moyens et les employés, le partage d'un même espace que seules les plantes vertes structurent, favoriseraient des rapports de convivialité.

Lc développement rapide des activités tertiaires oblige, dès 1960 , à produire des espaces de burcaux dont la conception doit satisfaire des exigences urbanistiques, économiques, organisationnelles et ... humaines. Le regroupement des sociétés se fera dans des tours implantées à la périphéric. Construire des plateaux ouverts coûte moins cher que de structurer l'espace par des cloisons et est plus adapté aux prévisions de restructuration, de flexibilité et de mobilité des activités, ainsi qu'à l'informatisation du travail et de la société.

La première réalisation des bureaux paysagers cut lieu en Allemagne par un groupe de consultants "Les Frères Schnclle" pour un grand groupe pharmaccutique. L'approche fut rapidement exportće aux Pays-Bas, en Scandinavie et en Grande-Bretagne, suivic par les États-Unis et par la France.

Dieter Jaeger, partner de "Quickborner tcam", dans la présentation qu'il fit aux États-Unis des concepts du système "office landscape" (1969 cité par B. Giraud, 1984), énonce le rapport entre sa conception du travail de bureau et sa conception des bureaux paysagers. Pour cet expert, les "office landscape systems" se sont développés "en premier lieu parce qu'ils étaient la solution pour rendre l'administration plus efficiente. En second lieu, l'aménagement de l'espace (paysagé, ouvert), peut combiner les éléments de ce système de traitement de l'information que sont les gens, les machines et autres équipements [...] l'immeuble est l'outil principal du travail administratif et du processus de décision [...] un bureau paysagé est comparable à un ordinateur non programmé et l'aménageur devient le véritable programmeur de cet espace-ordinateur; autrement dit, le véritable organisateur du travail. Enfin, par un 
plus grand contrôle des déplacements des personnels et un plus grand auto-contrôle de l'employé à son poste de travail, on peut obtenir un gain d'efficacité de 15\%".

Le point d'aboutissement de cette conception cybernétique de l'organisation du travail - et de son espace - comme un centre de traitement rationnel de l'information, serait l'exclusion de l'homme. En attendant que "l'organisation fonctionne optimalement comme un grand ordinateur, il faudra bien tenir compte de ces éléments du système de traitement de l'information" (Jaeger). La prise en compte obligée des besoins de l'homme au travail et que l'on peut instrumentaliser consistera alors à stimuler les sens par les couleurs, les plantes, la disposition du mobilier, l'éclairage... et à donner une certaine intimité afin que chacun puisse accomplir sa journée sans chute trop importante de son efficacité ; stimulation donnée pour compenser le caractère ennuyeux et répétitif $d u$ travail. De même, l'aménagement d'espaces de pauses doit permettre d'éviter que l'employé fasse semblant de travailler ou fasse des visites d'un bureau à l'autre sous couvert de prétextes de travail. Enfin, la saisie panoptique de tous les éléments qui concourent à la production du travail (postes de travail, objets, couleurs, circulation, bruit, lumière...) relève sans nul doute d'un besoin d'emprise totalitaire, revers d'une inclusion des personnels à un espace cybernétisé.

L'analyse des pratiques de ces espaces a mis en évidence l'utopie et le caractère idéologique de telles conceptions. D'une part, les relations de pouvoir ont retravaillé l'espace, tendant à recréer un marquage spatial de la hiérarchie, voire d'un recloisonnement généralisé, d'autre part, chacun a vécu cette ouverture spatiale comme perte de "privacy" et appauvrissement d'une vie relationnelle informelle. Dans un tel espace, compte tenu notamment de son caractère synoptique, les visites non nécessaires ou prolongées sont évitées ; la ponctualité et l'autodiscipline, l'ordre, la propreté, renforcés. La régulation par l'informel supprimé.

Nous sommes à l'opposé du plaisir et de la convivialité attendus. Les promesses de gain d'efficacité ou de productivité n'ont pas été tenues.

\section{Les stages d'intégration}

Les stages d'intégration réservés au jeunes cadres nouvellement embauchés ont pour objectif, par l'expérience vécue lors de ces stages, de favoriser l'identification à la culture de l'entreprise et de créer un sentiment d'appartenance. S'y rencontrent les jeunes cadres entrants, leurs futurs responsables, les "grands directeurs".

Le stage a généralement lieu dans une propriété prestigieuse. Alternent des activités de travail, de sport, des expériences de confrontation à un danger physique (quelque fois de survie), des activités artistiques, de loisirs, de farniente, de dynamique de groupe, de repas plus proches du festin que de la cantine.

L'apprentissage des normes de travail et de fonctionnement organisationnel est intégré à (enrobé par) l'expérience partagée de plaisir, de danger et de convivialité. La convivialité est la 
consigne donnée aux organisateurs de ces stages. L'expérience communautaire vécue lors de ces stages anticipe l'appréhension selon laquelle l'entreprise est une "communauté" et non, comme l'affirmait Max Weber, l'idéal-type d'une "société" structurée par la rationalité instrumentale. Entreprise qu'il conviendra d'investir selon le modèle du monde de la Science, de l'Art, du sport.

Simultanément à cette expérience d'intronisation, l'orientation professionnelle de chacun est préfigurćc par l'injonction : up ou out.

L'évolution du rapport au travail: la fragilisation identitaire

L'analyse de l'évolution sur vingt ans du rapport au travail et au sens du changement social, réalisée à partir de deux corpus d'entretiens qualitatifs (204 en 1977, 202 en 1997) appariés et redressés par rapport aux caractéristiques du travail et de l'emploi de chaque période, met en évidence le décalage radical entre le discours managérial que nous avons présenté ci-dessus et la réalité (objective et vécue) de la majorité des salariés, à l'exception de l'encadrement supérieur et des cadres moyens travaillant dans le tertiaire et susceptibles de devenir cadres supérieurs (J. Palmade, 2003).

Les sujets les plus affectés par les nouvelles conditions de travail et d'emploi (situation de précarité, menace de perdre l'emploi, déqualification, absence de progression professionnelle, mobilité contrainte, perte du métier...) sont essentiellement les employés et les ouvriers en incertitude d'emploi (intérim, CDD) et les cadres moyens, employés ou ouvriers en stabilité d'emploi mais déqualifiés.

La perte des attentes du travail, notamment sa valeur expressive et socialisatrice; l'absence d'appartenance à un collectif de travail spécifique des précaires, absence corrélative d'un affaiblissement d'intégration sociale, et d'un retrait du politique; le retrait (défensif) de l'investissement identitaire du travail et l'absence de confiance dans les acteurs du changement social (politiques, économiques, syndicaux, associatifs de la société civile); l'anxiété ou l'angoisse de l'avenir contribuent à une fragilisation identitaire corrélative d'un repli sur la sphère privée ou sur le Soi. À son tour, ce repli induit des conduites adaptatives de type "dépendance-soumission" aux conditions de travail, à l'Entreprise et au Pouvoir vécu comme anonyme et hégémonique, mais surtout à l'avenir dont il leur faut faire le deuil des promesses et des projets qui l'habitaient.

Le travail reste constitutif de l'identité et du lien social. L'emploi, devenu de plus en plus métonymie du travail, supprime la rétribution symbolique qu'apportent la reconnaissance des pairs et de la hiérarchic et la reconnaissance de la société en tant qu'il a une valeur d'utilité sociale, de production de l'homme.

Sur vingt ans, à l'exception de l'encadrement supérieur, on observe un processus de prolétarisation/précarisation, une dualisation entre l'encadrement supérieur et l'exécution. Le changement social ne profite pas de la même manière à tous.

Concernant les dimensions de sociabilité, 
convivialité, sentiment d'affiliation à un collectif de travail à la société, on observe un retrait très significatif des différentes composantes de l'affiliation sociale :

. un plus grand isolement au travail :

$+15 \%$

. une plus grande compétition, hostilité avec les collègues :

$+10 \%$

. une plus grande dépendance aux supérieurs :

$+20 \%$

. une plus grande psychologisation des

inégalités :

$+23 \%$

. une moins grande intégration sociale par

le travail :

$-16 \%$

. une plus grande résignation (dépendance au sens du changement social) :

$+14 \%$

- une moins grande délégation (aux acteurs du changement social) :

$-20 \%$

. un plus grand retrait politique :

$+20 \%$

Si la recherche dont nous venons de présenter les résultats les plus significatifs liés à la question examinée dans cet article, met en évidence un processus de désaffiliation (expression empruntée à $R$. Castel), d'autres recherches, conduites à la même période à partir d'autres méthodes d'investigation et portant sur de grands échantillons, aboutissent aux mêmes interprétations (Paugam, 2000 ; Baudelot et Gollac, 2003). C'est bien que ces résultats présentent une bonne robustesse et ont, en conséquence, valeur de généralisation.

À une absence d'intégration relationnelle/sociale au (et par le) travail, isolement que Serve Paugam qualifie "d'intégration professionnelle disqualifiante", correspond un retrait du politique.

\section{Sens du changement social et société conviviale ?}

La recherche déjà citée explorait les représentations et les attitudes vis-à-vis du sens du changement social, notamment le changement relatif à la communication, la sociabilité, l'intégration sociale.

L'analyse du sens de ce que disaient les interviewés dans les entretiens a mis en évidence, dès 1977 , une évaluation négative, négativité qui s'est très significativement accentuée en vingt ans (+20\%).

$1977 \quad 1998$

. absence de l'évocation

du thème

33

12

. évaluation positive

. évolution ambivalente

. évaluation négative

4

8

55

(précaires :

$80 \%)$

$100 \quad 100$

En 1998, à une question qui explorait à partir de 16 adjectifs les représentations sociales de la société, l'analyse des réponses (notes de 1 à 7) met en évidence l'importance des connotations négatives. Les représentations sont cependant spécifiques des groupes sociaux. 
Représentations de la société (échantillon redressé, 1998)

$\begin{array}{lll}\text { inquiétante } & 71 \% & \\ \text { explosive } & 58 \% & \text { Toute-puissance } \\ \text { implacable } & 57 \% & 45 \% \\ \text { vide } & 57 \% & \end{array}$

$\begin{array}{ll}\text { créatrice } & 38 \% \\ \text { conquérante } & 31 \% \\ \text { vivifiante } & 20 \% \\ \text { libératrice } & 6 \%\end{array}$

les cadres de direction :

les travailleurs indépendants (incertains) :

conquérante

libératrice

toute-puissante

vivifiante

créatrice

créatrice

l'exécution stable :

l'exécution incertains $>35$ ans :

inquiétante

implacable

implacable

explosive

les intérimaires :

syndicalistes, associatifs

(opposés à l'économie

néo-libérale) :

vide

explosive 
L'ensemble de ces résultats met en évidence le caractère d'idéalité d'une société conviviale, unifiée, maître de son avenir.

On observe plusieurs niveaux de dualisation de groupes de sujets différenciés selon plusieurs critères : l'adhésion, le refus ou la soumission à l'économie libérale ; le statut dans l'emploi ; la probabilité différentielle de perdre l'emploi ; la place dans la division du travail ; l'âge selon le sexe.

Aujourd'hui, la Société est multi-duale, les sujets de plus en plus isolés. La qualité de l'ambiance au travail, le besoin d'appartenance à un collectif stable, restent des valeurs essentielles. Elles sont cependant significativement différentielles selon les catégories d'acteurs.

La convivialité est une des composantes de l'ambiance, surtout pour les femmes employées et les hommes de moins de 35 ans en situation de précarité d'emploi.

Convivialité n'est pas solidarité.

\section{R É $\quad$ F}

ABÉCASSIS F., ROCHE P. (2001), Précarisation du travail et lien social. Des hommes en trop, Paris, L'Harmattan.

BARTOLI A. (1990), Communication et organisation, Paris, Les Éditions d'Organisation. BAUDELOT Ch. GOLLAC M. (2003), Travailler pour être heureux? Le bonheur et le travail, Paris, Fayard.

BOLTANSKI L., CHIAPPELLO E. (1999), Le nouvel esprit du capitalisme, Paris, NRF, Gallimard.

DESJOURS Ch. (1993), Travail usure mentale, de la psychopathologie à la psychodynamique du travail, Paris, Fayard.

DULUC A. (2003), Leadership et confiance: développer le capital humain pour des organisations performantes, Paris, Dunod.

GIRAUD B. (1984), Quelle psychosociologie pour l'espace de travail ? Le cas des bureaux. Thèse de sociologie, Université Paris-Dauphine.

KARASEK R., TÖRES Th. (1990), Healthy work: stress, productivity, and the reconstruction of the working life, New York, Basic Book.

JAEGER D. (1969), Office Landscape, a systems concept, Ed. The business press, Elmhurst, Illinois, USA.

PALMADE J. (dir) (2003), L'incertitude comme norme, Paris, PUF.

PAUGAM S. (2000), Le salarié de la précarité, Paris, PUF.

SALMON A. (2002), Ethique et ordre économique. Une entreprise de séduction, Paris, CNRS, Sociologie.

WESTPHALEN M. H. (1989), Le communicator, Paris, Dunod. 